\title{
Effectiveness of Porcelain Veneer on Dental Restoration in Rats with Periodontitis
}

\author{
SHUANG LIU*, HUI YAN AND YUEHANG GAO \\ Department of Stomatology, Nankai Hospital, Tianjin 300100, China
}

Liu et al.: Porcelain Veneer on Dental Restoration in Rats

\begin{abstract}
The purpose of this study is to explore the effectiveness of porcelain veneer technology on periodontitis in rats, so as to better guide clinical practice. Using 70 rats the periodontitis rat model was established. Porcelain veneer was used to repair the rat teeth. Follow-up evaluation of the curative effect was carried out at 2, 4, 6 and 8 weeks after operation, so as to gain a certain understanding of the treatment of periodontitis with porcelain veneer. The results showed that after porcelain veneer restoration, the teeth of periodontitis rats constructed by string ligation became wider, the space between teeth become smaller, the height of tooth bone decreased and the teeth were closely adhered to the gingiva. After operation, alveolar bone volume significantly increased $(\mathbf{P}<\mathbf{0 . 0 5})$. The follow-up results showed that the success rates of porcelain veneer were $100,100,97.5$ and $95 \%$ on the $2,4,6$ and 8 week after operation. These results indicated that the ceramic veneer exerted a good restorative effect on rat teeth with periodontitis and has a high stability after operation. It is hence worth further investing future in clinical research
\end{abstract}

Key words: Porcelain veneer, rats with periodontitis, dental restoration, therapeutic effect tracking

Periodontitis is a common oral disease caused by bacterial biofilm infection of dental plaque. This biofilm leads to the formation of periodontal pockets, and develops into inflammation-mediated loss of connective tissue attachment and alveolar bone destruction, which eventually leads to tooth $\operatorname{loss}^{[1]}$. The condition of periodontitis will aggravate with the increase of age and recover after treatment. The prevalence of periodontitis in the world is about 5-20\%, and people of all ages may be infected ${ }^{[2,3]}$. In recent years, the incidence of periodontitis in China is increasing. Porcelain veneer has been used for many years as a restorative material. It combines bonding material with tooth surface tissue to protect periodontal tissue from damage ${ }^{[4]}$. With the continuous improvement of ceramic veneer restoration technology, the preparation of ceramic veneer teeth, the bonding stability and the degree of appearance beautification have made a great leap, which has been widely used in the field of dental restoration ${ }^{[5]}$. Porcelain veneer repair methods can be divided into direct veneer repair and indirect veneer repair, mostly ceramic materials. Because the prosthesis sometimes falls off and discoloration during occlusion and chewing after porcelain veneer restoration, it is usually necessary to have a follow-up visit within a certain time ${ }^{[6]}$. In the study of periodontal disease, animal models are very important for studying the relationship between disease and external factors and 
testing potential new therapies. These can be used to construct periodontal lesion models, such as connective tissue destruction, bone loss and periodontal pocket formation $^{[7]}$. Since collagen degradation and gingival tissue structure has been observed in alveolar bone loss in rats and humans, rats are the most widely used rodents to study the pathogenesis of periodontal disease ${ }^{[8]}$. At present, there are many ways to induce periodontitis in rats, but ligation of mandibular molars with thin rope is a simple and easy method ${ }^{[9]}$. Radiography, morphometry and histology are the main techniques used to quantify the severity of periodontitis, regardless of the method used to induce periodontitis. Although there have been some studies on the treatment of periodontitis in rats, there are few reports on the restoration of rat teeth with porcelain veneer. Therefore, in this study, rats with periodontitis were used as the test system to evaluate the efficacy of porcelain veneer in the treatment of periodontitis, hoping to provide a new way for the treatment of periodontitis. Vita 3D Master colorimetric board (Fengda Dental Equipment Co., Ltd., China) was used to compare the dental surface of rats and select suitable veneer color. The model of base toothpaste and veneer wax were made and put into an electric oven (Guangzhou Zhanzhuo Commercial Equipment Manufacturing Co., Ltd., China) to make the body porcelain back-cut after refractory sintering and natural cooling. According to the colorimetric results, the corresponding veneer is selected. The restorations are dyed with color porcelain powder and covered with cut-end magnets and body ceramics. The restorations were pickled with 35\% phosphoric acid (Wuhan Yixing Da Chemical Co., Ltd., China) for 2 min, rinsed and dried. Seventy adult rats (Shanghai Yisen Biotechnology Co., Ltd., China) weighing 310$330 \mathrm{~g}$ were purchased. The rats were housed in controlled atmosphere of $23^{\circ}, 55 \%$ humidity $12 \mathrm{~h}$ of dark and light cycle for $2 \mathrm{w}$. Animal experimental protocols were approved by Ethics Committee of Nankai Hospital. The rats were randomly divided into 3 groups, control group (20 rats), $5 \mathrm{~d}$ ligation group (25 rats) and $10 \mathrm{~d}$ ligation group (25 rats). Eight hours before the experiment rats were not allowed access to water. Rats are anesthetized with $0.5 \mathrm{ml} / 100 \mathrm{~g}$ dose of $7 \%$ chloral hydrate (Yinhai Chemical Group Co., Ltd., China), limbs were fixed on the platform abdomen upward and the upper and lower teeth were pulled with a thin rope and fixed. In the space between molars, the filaments are wounded and ligated. A tooth hook was used to separate the gingiva and tweezers were used to clamp the ligating wire and tie it tightly in the cervical buckle to cut off and close the truncation. After feeding, rats were monitored continuously. Development of periodontitis was validated on $\mathrm{d} 0,5$ and 10 after operation. Three rats in each group were randomly selected. Gingival index, periodontal pocket depth, tooth mobility and alveolar bone resorption were measured with periodontal probe (Xiamen Yushang Hardware Tools Co., Ltd., China), and its average value was recorded. After testing, the mandible was separated and fixed in $5 \%$ polyformaldehyde (Yangzhou Zhongjiang Material Technology Co., Ltd., China) for $2 \mathrm{~d}$. The mandible was decalcified in formic acid (Beijing Baiolaibo Technology Co., Ltd., China). After dehydration, it was embedded in paraffin and thin tissue sections were prepared. The periodontal pathological changes were observed under an optical microscopy (Nikon, Japan) after staining with hematoxylin-eosin (Shanghai Meixuan Biotechnology Co., Ltd., China). Porcelain veneer was used to restore the teeth of periodontitis rats. Firstly, porcelain veneer restorations were treated with $10 \%$ hydrofluoric acid (Jinan Baike Chemical Co., Ltd., China). After rinsing, soaked for $3 \mathrm{~min}$ in 95\% medical alcohol (Guangxi Boheng Medical Products Co., Ltd., China) and then coated with silane coupling agent (Guangzhou Yuantai Synthetic Materials Co., Ltd., China). After $1 \mathrm{~min}$, it was dried and finally coated with resin cement (Coltene, Switzerland). Pumice powder solution (Suzhou Zhenglong Trading Co., Ltd., China) was used to clean the affected teeth of rats with periodontitis, and then $35 \%$ phosphoric acid (Wuhan Yixing Da Chemical Co., Ltd., China) was used to corrode the teeth for $20 \mathrm{~s}$. After smearing the adhesive, it was dried. The restorations were attached to the affected teeth, where the cement was solidified by light, and the cement was completely fixed by applying an oxygen-insulating agent (Ivoclar, Liechtenstein) on the edge of the adhesive. Four rats were randomly selected, sacrificed, the jaw bone was removed and scanned by high resolution micro-CT (Shunyun Instrument Co., Ltd., China). The changes of alveolar bone in rats before and after operation were observed and transformed into data analysis. All rats with periodontitis repaired with porcelain veneer were fed normally after operation, and were reexamined after 2, 4, 6 and $8 \mathrm{w}$. The evaluation criteria were shown in Table 1 . The effect of porcelain veneer restoration was evaluated according to 3 grades, excellent, good and poor. Excellent and good were regarded as success while poor was failure. SPSS22.0 software was used to statistics the experimental results, and the data are expressed as mean \pm standard deviation Comparison 
between groups was based on one-way variance. When $\mathrm{P}<0.05$, the difference is statistically significant. Qualitative data are expressed in $\mathrm{n}(\%)$. The periodontal tissues of experimental rats are shown in fig. 1. Before ligation, the gingival surface of rats was intact and closely attached to the tooth surface. A small number of inflammatory cells infiltrated the connective tissue and the alveolar bone was intact. Five $d$ after ligation of molars, the surface of gingiva was ulcerated. A large number of scales and calculi appeared at the ligation site, and the color of gingiva was dark. There were obvious signs of bleeding, and periodontal pockets formed. After $10 \mathrm{~d}$ of ligation, the periodontal pockets were obviously deepened, the root of the experimental teeth was exposed and very loose, the gingival papilla was destroyed, and the alveolar bone resorption led to the reduction of alveolar bone height. As shown in
Table 2, the gingival index, periodontal pocket depth, tooth mobility and alveolar bone resorption in 5-d group and 10-d group were significantly higher than those in the control group, indicating that the ligation experiment had an impact on rat teeth. The above results showed that the periodontitis model of experimental rats was successful, and the rats ligated for 5 and $10 \mathrm{~d}$ can be used for follow-up experiments.

From the three-dimensional images taken, it can be seen from fig. 2 that the teeth of periodontitis rats were narrower, the height of the teeth was more, the roots were exposed, and there were obvious spaces between the gingiva and the teeth. After porcelain veneer treatment, the tooth surface of rats became wider, the adjacent teeth were arranged tightly, the height of the tooth bone decreased, and the gum was tightly adhered. After calculating the alveolar bone volume of rats with

TABLE 1: EVALUATION CRITERIA FOR PORCELAIN VENEER RESTORATION

\begin{tabular}{|c|c|c|c|}
\hline \multirow{2}{*}{ Assessment projects } & \multicolumn{3}{|c|}{ Grade } \\
\hline & Excellent & Good & Poor \\
\hline $\begin{array}{l}\text { Tidiness of porcelain } \\
\text { veneer }\end{array}$ & Complete & $\begin{array}{l}\text { There are some defects, but they do } \\
\text { not affect the beauty. }\end{array}$ & There are some cracks, breaks or s \\
\hline Edge fitness & $\begin{array}{l}\text { Complete, } \\
\text { seamless fit }\end{array}$ & $\begin{array}{l}\text { There are some small gaps where the } \\
\text { probe can enter. }\end{array}$ & $\begin{array}{l}\text { The crack is obvious and the tooth surface } \\
\text { is exposed. }\end{array}$ \\
\hline $\begin{array}{l}\text { Porcelain veneer } \\
\text { color }\end{array}$ & No discoloration & There is a slight discoloration. & Obvious discoloration \\
\hline Dental caries & No dental caries & There are superficial caries. & Deep caries \\
\hline $\begin{array}{l}\text { Postoperative } \\
\text { sensitivity }\end{array}$ & $\begin{array}{l}\text { Insensitive } \\
\text { reaction }\end{array}$ & $\begin{array}{l}\text { Mild sensitivities disappear after } \\
\text { wearing teeth. }\end{array}$ & Very sensitive, tooth inflammation. \\
\hline Gingival abnormality & No abnormality & $\begin{array}{l}\text { There is mild inflammation and a } \\
\text { small amount of bleeding. }\end{array}$ & $\begin{array}{l}\text { There is marked redness and swelling and } \\
\text { periodontal pocket formation. }\end{array}$ \\
\hline
\end{tabular}

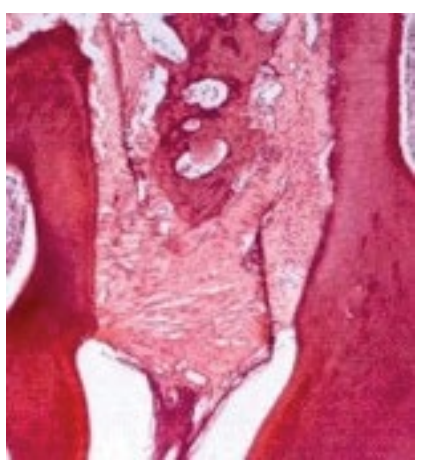

A

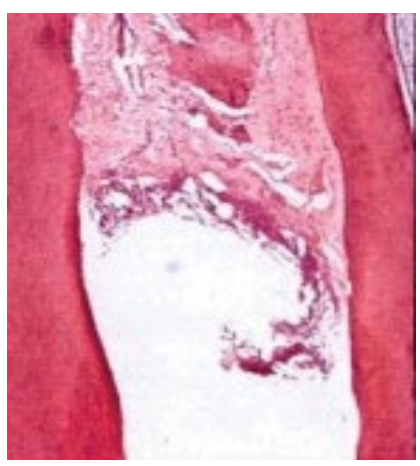

B

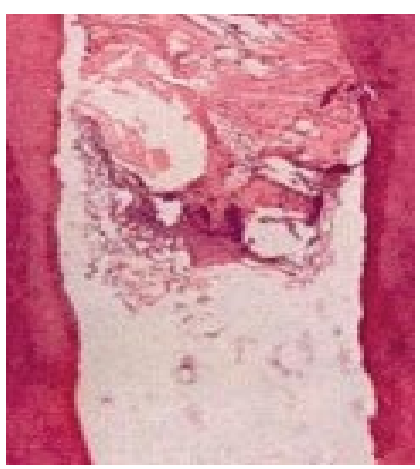

C

Fig. 1: Histopathology of periodontal tissue in experimental rats

TABLE 2: GINGIVAL INDEX, PERIODONTAL POCKET DEPTH, TOOTH MOBILITY AND ALVEOLAR BONE RESORPTION OF RATS IN EACH GROUP

\begin{tabular}{lccc}
\hline & Control group & 5-day ligation group & 10-day ligation group \\
\hline Gingival index & $0.00 \pm 0.00$ & $2.03 \pm 0.41$ & $2.16 \pm 0.20$ \\
Periodontal pocket depth $(\mathrm{mm})$ & $0.21 \pm 0.08$ & $3.13 \pm 0.24$ & $3.35 \pm 0.21$ \\
Tooth mobility & $0.00 \pm 0.00$ & $1.28 \pm 0.35$ & $1.35 \pm 0.36$ \\
Alveolar bone resorption $(\mathrm{mm})$ & $0.00 \pm 0.00$ & $0.75 \pm 0.09$ & $2.02 \pm 0.13$ \\
\hline
\end{tabular}

There is a significant difference between the control group and the 5-day ligation group $(P<0.05)$, while there is a significant difference between the control group and the 10-day ligation group $(P<0.05)$. 
periodontitis before and after porcelain veneer treatment was compared with that before treatment as shown in fig. 3. After treatment, the alveolar bone volume increased significantly $(\mathrm{P}<0.05)$. The results showed that porcelain veneer can effectively increase the bone volume of rats with periodontitis. The rats with periodontitis at 2, 4, 6 and 8 weeks after operation were followed up and evaluated. The results are shown in Table 3. In terms of the regularity of porcelain veneer, $38,38,37$ and 37 rats with periodontitis have complete porcelain veneer 2, 4, 6 and $8 \mathrm{w}$ after operation, and 2, 2, 2 and 1 rats showed defective teeth that did not affect the appearance. At 6 and $8 \mathrm{w}, 1$ or 2 rats porcelain veneers were broken and needed to be repainted and pasted. In terms of marginal fitness, 39, 39, 38 and 38 rats with periodontitis at 2, 4, 6 and $8 \mathrm{w}$ after operation can seamlessly fit the edges, but the probes cannot penetrate into the edges. Small gaps appeared at the edges of 1, 1, 2 and 2 rats, which can clamp the sharp probes. As for the color of porcelain veneer, it kept

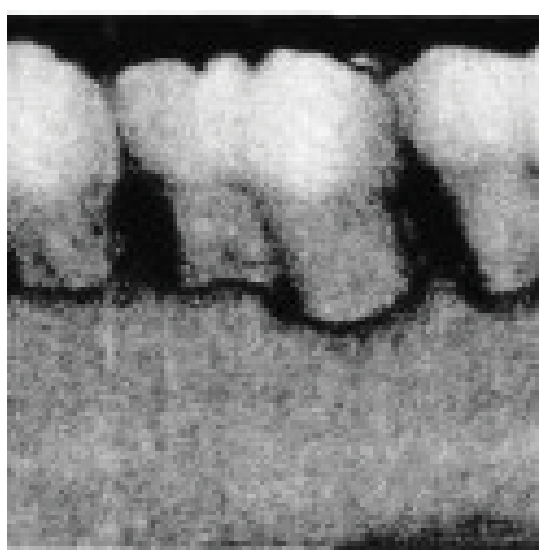

A

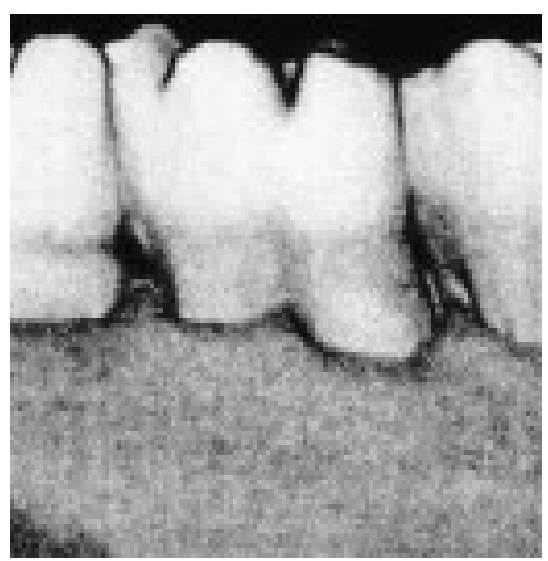

B

Fig. 2: Microscopic CT three-dimensional image of alveolar bone in rats

TABLE 3: ASSESSMENT RESULTS OF PORCELAIN VENEER REPAIR

\begin{tabular}{|c|c|c|c|c|}
\hline \multirow{2}{*}{ Assessment project } & \multicolumn{4}{|c|}{ Assessment time } \\
\hline & 2 weeks $(n=40)$ & 4 weeks $(n=40)$ & 6 weeks $(n=40)$ & 8 weeks $(n=40)$ \\
\hline \multicolumn{5}{|c|}{ Tidiness of porcelain veneer } \\
\hline Excellent & $38(95)$ & $38(95)$ & $37(92.5)$ & $37(92.5)$ \\
\hline Good & $2(5)$ & $2(5)$ & $2(5)$ & $1(2.5)$ \\
\hline Poor & 0 & 0 & $1(2.5)$ & $2(5)$ \\
\hline \multicolumn{5}{|l|}{ Edge fitness } \\
\hline Excellent & $39(97.5)$ & $39(97.5)$ & $38(95)$ & $38(95)$ \\
\hline Good & $1(2.5)$ & $1(2.5)$ & $2(5)$ & $2(5)$ \\
\hline Poor & 0 & 0 & 0 & 0 \\
\hline \multicolumn{5}{|l|}{ Porcelain veneer color } \\
\hline Excellent & $40(100)$ & $40(100)$ & $39(97.5)$ & $39(97.5)$ \\
\hline Good & 0 & 0 & $1(2.5)$ & $1(2.5)$ \\
\hline Poor & 0 & 0 & 0 & 0 \\
\hline \multicolumn{5}{|l|}{ Dental caries } \\
\hline Excellent & $40(100)$ & $40(100)$ & $40(100)$ & $39(97.5)$ \\
\hline Good & 0 & 0 & 0 & $1(2.5)$ \\
\hline Poor & 0 & 0 & 0 & 0 \\
\hline \multicolumn{5}{|l|}{ Postoperative sensitivity } \\
\hline Excellent & $38(95)$ & $39(97.5)$ & $40(100)$ & $40(100)$ \\
\hline Good & $2(5)$ & $1(2.5)$ & 0 & 0 \\
\hline Poor & 0 & 0 & 0 & 0 \\
\hline \multicolumn{5}{|l|}{ Gingival abnormalities } \\
\hline Excellent & $38(95)$ & $38(95)$ & $39(97.5)$ & $39(97.5)$ \\
\hline Good & $2(5)$ & $2(5)$ & $1(2.5)$ & $1(2.5)$ \\
\hline Poor & 0 & 0 & 0 & 0 \\
\hline
\end{tabular}




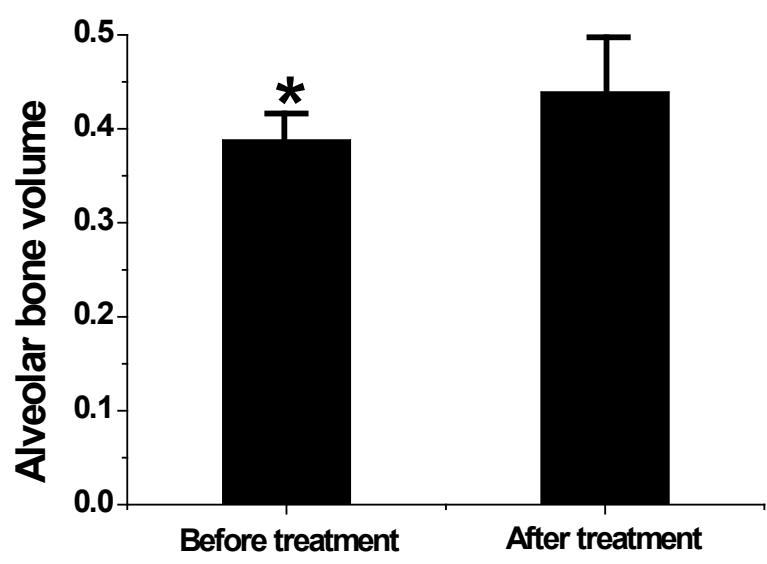

Fig. 3: Comparison of alveolar bone volume before and after treatment with porcelain veneer in rats with periodontitis

good basically. Only 1 or 2 rats with periodontitis show slight discoloration in 6 and $8 \mathrm{w}$. As for caries occurrence, only one case has mild secondary caries at $8 \mathrm{w}$. Postoperative sensitivity was observed in 38 and 39 patients at 2 and $4 \mathrm{w}$, respectively. No significant sensitivities were observed in 2 and 1 patients. With the passage of time, no postoperative sensitivity occurs. As for gingival abnormalities, 38, 38, 39 and 39 periodontitis rats have no gingival abnormalities at 2, 4, 6 and $8 \mathrm{w}$ postoperatively, while only gingivitis and a little bleeding were found at 2, 2, 1 and $1 \mathrm{w}$ postoperatively. The success rate of porcelain veneer is $100,100,97.5$ and $95 \%$ at 2, 4, 6 and $8 w$ after operation. At present, the treatment of periodontitis is mainly divided into traditional Chinese medicine and Western medicine. Traditional Chinese medicine relieves periodontal symptoms mainly by nourishing kidney and pulp, clearing away heat and purging fire, while western medicine cures periodontitis by curettage, cleaning periodontitis and taking antibiotics. Traditional Chinese medicine treatment course is long and easy to relapse. Western medicine treatment process is complex and not conducive to beautiful teeth. Porcelain veneer can effectively reduce the degree of tooth damage, and has cosmetic effect, which can be gradually promoted in clinical use. In this study, the experimental model of periodontitis in rats is established, and the restoration technology of porcelain veneer is used to treat tooth injury. The regularity, marginal fitness, color of porcelain veneer, occurrence of dental caries, postoperative sensitivity and gingival abnormality are evaluated and counted regularly after operation. The results show that the porcelain veneer can effectively increase the volume of alveolar bone, reduce the space between teeth, reduce the height of alveolar bone, and enhance the bonding degree between alveolar bone and gingiva. At the same time, the porcelain veneer has strong stability and affinity, and few changes or adverse tooth reactions occur after operation. In summary, in order to study the clinical effect of porcelain veneer in the restoration of periodontitis rat teeth, the technology of porcelain veneer is used to treat periodontitis rat teeth and monitor its prognosis, and the results are as expected. This study provides experimental data for the clinical application of porcelain veneer in the treatment of periodontitis. However, there are still some shortcomings in this study, such as a small number of experimental samples, inadequate monitoring and evaluation indicators after surgery. Therefore, followup studies still need to increase the sample size, constantly improve the mechanism of post-operative evaluation and do in-depth research at the molecular level.

\section{REFERENCES}

1. Tonetti MS, Chapple IL, Jepsen S. Primary and secondary prevention of periodontal and peri-implant diseases: Introduction to, and objectives of the 11th European Workshop on Periodontology consensus conference. J Clin Periodontol 2015;16(S16):1-4.

2. Perez LA, Al-Shammari KF, Giannobile WV. Treatment of Periodontal Disease in a Patient With Ehlers-Danlos Syndrome. A Case Report and Literature Review. J Clin Periodontol 2017;73(5):564-70.

3. Eke PI, Dye BA, Wei L. Prevalence of periodontitis in adults in the United States: 2009 and 2010. J Dent Res 2012;91(10):91420.

4. Cunha LFD, Pedroche LO, Gonzaga CC. Esthetic, occlusal, and periodontal rehabilitation of anterior teeth with minimum thickness porcelain laminate veneers. Br Dent $\mathrm{J}$ 2014;112(6):1315-18.

5. Lee HS, Kwon TY. The Application of a Novel Ceramic Liner Improves Bonding between Zirconia and Veneering Porcelain. Materials 2017;10(9):1023.

6. Hong N, Yang H, Li J. Effect of Preparation Designs on the Prognosis of Porcelain Laminate Veneers: A Systematic Review and Meta-Analysis. Oper Dent 2017;42(6):E197.

7. Aguirre JI, Akhter MP, Kimmel DB, Pingel J, Xia X, Williams A. Enhanced alveolar bone loss in a model of non-invasive periodontitis in rice rats. Oral Dis 2012;18:459-68.

8. Struillou X, Boutigny H, Soueidan A, Layrolle P. Experimental animal models in periodontology: a review. Open Dent J 2010.

9. Zhang W, Ju J, Rigney T, Tribble G. Porphyromonas gingivalis infection increases osteoclastic bone resorption and osteoblastic bone formation in a periodontitis mouse model. BMC Oral Health 2014;14:89.

This is an open access article distributed under the terms of the Creative Commons Attribution-NonCommercial-ShareAlike 3.0 License, which allows others to remix, tweak, and build upon the work non-commercially, as long as the author is credited and the new creations are licensed under the identical terms

This article was originally published in Special issue on "Trends in therapeutic Management of Various Conditions" Indian J Pharm Sci 2020:82(3) spl issue 6;92-96 